\title{
Transmission Efficiency of Papaya ringspot virus by Three Aphid Species
}

\author{
C. M. Kalleshwaraswamy and N. K. Krishna Kumar
}

First author: Research scholar, Division of Entomology and Nematology, Indian Institute of Horticultural Research, Bangalore 560 089, India; second author: Principal Scientist \& Head, Division of Entomology and Nematology, Indian Institute of Horticultural Research, Bangalore 560 089, India.

Accepted for publication 13 September 2007.

\begin{abstract}
Kalleshwaraswamy, C. M., and Krishna Kumar, N. K. 2008. Transmission efficiency of Papaya ringspot virus by three aphid species. Phytopathology 98:541-546.

The transmission efficiency of Papaya ringspot virus (PRSV) by three aphid vectors (i.e., Aphis gossypii, A. craccivora, and Myzus persicae) was studied. Efficiency was measured by single-aphid inoculation, group inoculation (using five aphids), duration of virus retention, and the number of plants following a single acquisition access period (AAP) to which the aphids could successfully transmit the virus. Single-aphid inoculation studies indicated that $M$. persicae (56\%) and A. gossypii (53\%) were significantly more efficient in transmitting PRSV than A. craccivora $(38 \%)$. Further, in the former two species, the time required for initiation of the first probe on the inoculation test plant was significantly shorter compared to A. craccivora. PRSV transmission efficiency was $100 \%$ in all three species when a group of five aphids were used per

sequestration period increased, although $M$. persicae successfully transmitted PRSV after $30 \mathrm{~min}$ of sequestration. A simple leaf-disk assay technique was employed for evaluating the transmission efficiency of three species of aphids. The results of leaf-disk assays also indicated that A. gossypii (48\%) and M. persicae (56\%) were more efficient PRSV vectors than $A$. craccivora. Using leaf-disk assays, the ability of individual aphids to inoculate PRSV serially to a number of plants was studied. Following a single AAP on an infected leaf, M. persicae was more efficient than the other two species with $52.5 \%$ transmission after the first inoculation access period (IAP). However, its inoculation efficiency significantly decreased with the second and subsequent IAPs. $A$. gossypii was able to transmit PRSV sequentially up to four successive leaf disks, but with significantly declining efficiency. Since A. gossypii is reported to be the numerically dominant vector in south India in addition to being a more efficient vector capable of inoculating PRSV to multiple plants, it should be the target vector for control strategies.
\end{abstract} plant. There was a perceptible decline in transmission efficiency as the
Papaya (Carica papaya L.), an important fruit crop of India, is extensively cultivated in south India and parts of north India (Fig. 1). In the last decade, the area under papaya cultivation in India has dramatically increased. Following liberalization and introduction of some superior Taiwanese and Hawaiian varieties/hybrids, papaya cultivation has increased. A major setback subsequent to introduction of exotic varieties has been the occurrence of Papaya ringspot virus (PRSV). PRSV was first reported from Uttar Pradesh (9) and thereafter from all the north Indian states. However, PRSV was not reported until 1995 from south India (1). Within 5 years, PRSV had spread all over south India causing significant yield loss (17).

PRSV (family Potyviridae, genus Potyvirus) is transmitted by several species of aphids in a nonpersistent manner (2). This type of transmission is generally characterized by a short acquisition access period (AAP) of a few seconds to minutes, absence of a distinct latent period, and a short inoculation access period (IAP) (23). Thus, the virus is acquired during brief exploratory probes in the process of host finding and is transmitted to a healthy papaya plant within a few seconds to minutes. Aphids do not normally colonize papaya and natural transmission is reported to occur through transient aphid vectors $(8,10)$. Several aphid species such as Aphis nerii Boyer de Fonscolombe, A. gossypii Glover, A. spiraecola Patch, Myzus persicae (Sulzer), Toxoptera aurantii (Boyer de Fonscolombe), A. craccivora Koch, and Rhopalo-

Corresponding author: N. K. Krishna Kumar; E-mail address: nkkumar@iihr.ernet.in

doi:10.1094/PHYTO-98-5-0541

(C) 2008 The American Phytopathological Society siphum maidis (Fitch) are reported as PRSV vectors $(11,16,20)$. PRSV is the most widespread and damaging viral disease affecting papaya production in many parts of the world. Plants of all ages are vulnerable to PRSV. Affected plants become stunted leading to reduction in fruit set (2). PRSV-P (papaya biotype) infects papaya and cucurbits, while, PRSV-W (watermelon biotype) is restricted only to cucurbits (14).

Though genetically modified PRSV resistant papaya varieties have been reported (22), differences in coat protein sequences of Indian isolates indicate need for strain-specific transgenic papaya (4). In the absence of a durable PRSV resistant papaya cultivar/hybrid, farmers often resort to indiscriminate application of insecticides to reduce yield loss. This could be futile in the absence of a scientific understanding of the role of aphid vectors in the primary and secondary spread influencing PRSV epidemiology. Our earlier studies in papaya orchards using yellow funnel water traps indicated that A. gossypii is one of the predominant vectors, followed by $A$. craccivora and $M$. persicae (8). Peak aphid population of $A$. gossypii during March and April was a primary factor for a high incidence of new PRSV infections during April to May (8). The seasonal dynamics of A. gossypii was linked to the large scale cultivation of cucurbitaceous vegetables from November to late March in south India (8). However, no information is available on the PRSV transmission efficiency of these vectors. Besides aphid number, a number of vector mediated components contribute to PRSV transmission efficiency. These include the innate efficiency of individuals within the aphid population to transmit PRSV, ability to retain the virus following acquisition and last but not least, the ability to infect a series of susceptible host plants following single acquisi- 
tion. The aim of this study was to identify the transmission efficiency through single and group aphid inoculation, probing behavior, duration of PRSV retention following AAP and ability to transmit to a series/number of papaya plants across three vector species viz. A. gossypii, A. craccivora, and $M$. persicae. The importance of vector number and transmission efficiency in the epidemiology of PRSV is discussed.

\section{MATERIALS AND METHODS}

Maintenance of aphid colonies, virus inoculum, and test plants. Aphis gossypii were collected on eggplant (Solanum melongena L.) leaves and reared on cotton (Gossypium hirsutum L., $D C H$-32) seedlings inside an insect-proof cage $(50 \times 50 \times 50$ $\mathrm{cm}) \mathrm{kept}$ in a glass house. A. craccivora and $M$. persicae were maintained on cowpea (Vigna unguiculata L., var. C-152) and radish (Raphanus sativus L., var. Arka Nishant), respectively. For maintenance of virus inoculum, young terminal leaves were collected from plants (approximately 12 months old) suspected to be infected with PRSV-P. Leaf samples were subjected to reverse transcription polymerase chain reaction (RT-PCR) using PRSVcoat protein (cp) specific primers PRSV Bam 8 (F): 5'-AAA GGA TCC ATG GGT TTG AAT GAG AAG TTG AAG A-3' and PRSV Bam 8 (R): 5'-AAT GGA TCC AAT GCG CAT ACC CAA GA-3' to confirm that the plants were infected by PRSV. Virus inoculum from RT-PCR positive plants was mechanically inoculated to 60-day-old papaya seedlings (hybrid Surya). Inoculated plants were maintained in an aphid-proof nylon mesh cage and used as virus source-plants for transmission studies. As test plants, papaya (hybrid Surya) seedlings were raised in $150 \mathrm{cc}$, polyethylene cups inside an aphid-proof nylon net (40 mesh). Seedlings with two to three pairs of fully opened leaves (60 days old) were used for virus inoculation in all transmission experiments.

DAC-ELISA. To confirm PRSV infection, apical leaves were collected from each test plant separately for direct antibody coating-enzyme linked immunosorbent assay (DAC-ELISA) (5). The polyclonal antibody of PRSV was obtained from Vittal Mallya Research Foundation, Bangalore, India. Absorbance values were recorded at $405 \mathrm{~nm}$ using an ELISA reader

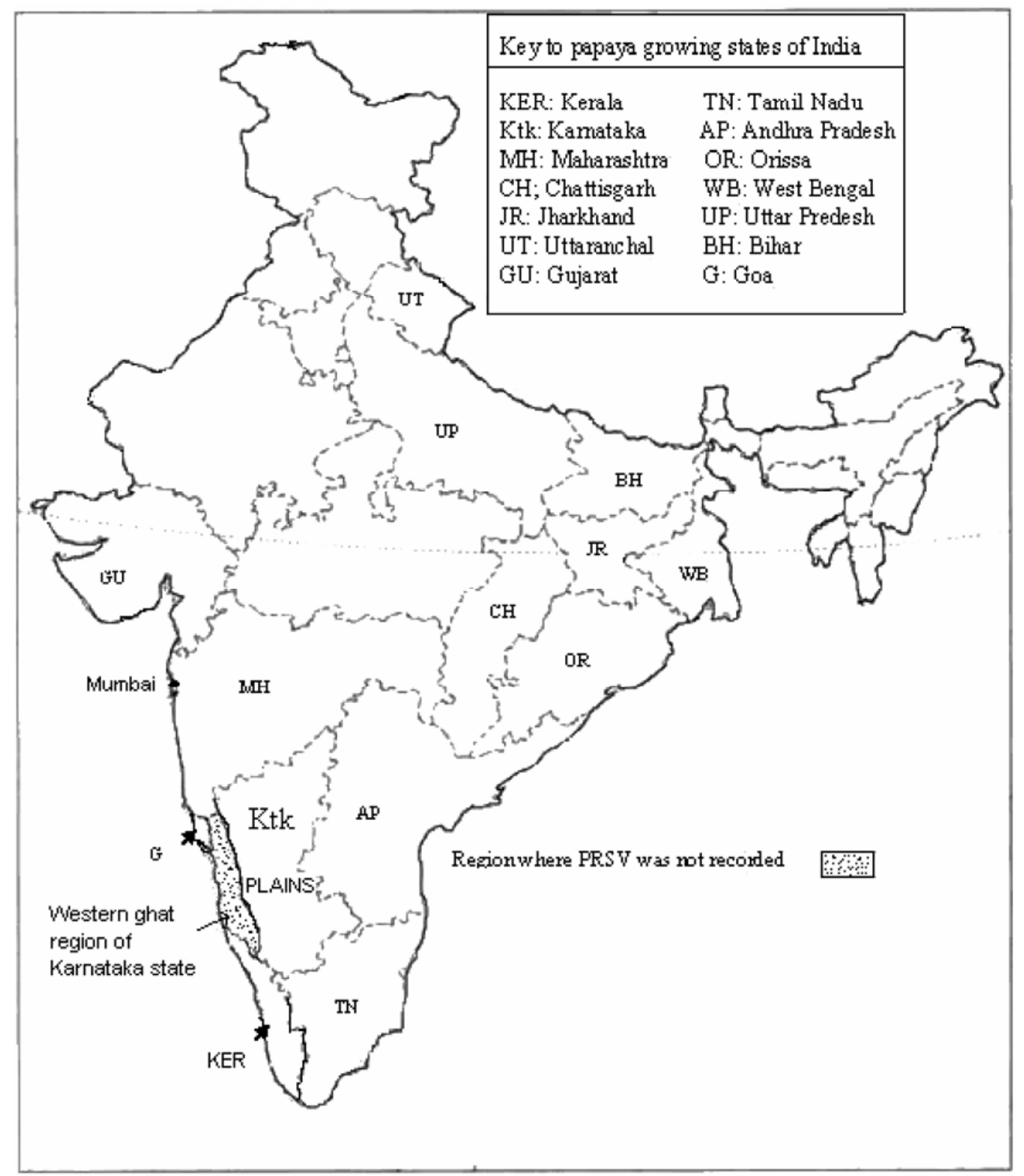

Fig. 1. States of India where papaya is cultivated commercially and western ghat region (represented in dots) of Karnataka state where Papaya ringspot virus (PRSV) was not recorded. 
(Multiskan EX model 352). Samples were considered positive if the ELISA readings were $>3$ times the mean of healthy papaya leaf samples.

Measurement of vector efficiency. Single aphid inoculation. One day prior to transmission, late instar nymphs expressing wing buds were confined to a petri dish containing a healthy leaf of a respective host plant. Alate aphids that emerged $24 \mathrm{~h}$ later were selected for transmission studies. Prior to the acquisition access period (AAP), aphids were starved for $2 \mathrm{~h}$. Individual aphids were placed on PRSV infected source leaves and observed for probing using a hand lens $(10 \times)$. When an aphid pressed its labium to the plant surface and moved its antennae back across the dorsum, it was considered to be probing. AAP was considered to begin with the first probe, after which aphids were allowed to remain on the leaf for $5 \mathrm{~min}$. After this AAP, the aphid was transferred from the virus source leaf using a fine paint brush to the first apical leaf of a healthy, 60-day-old papaya seedling for an inoculation access period (IAP) of $5 \mathrm{~min}$.

On each inoculation test plant, the time required for the initiation of the first probe (the time elapsed after the aphid was placed on the plant to the time the aphid began probing) was recorded. Mean time spent to initiate probing on the inoculation test plant was compared using analysis of variance (ANOVA). IAP was considered to have started with the first probe, following which aphids were allowed to probe for $5 \mathrm{~min}$. After the IAP, aphids were removed from the test plant. In this way, 25 papaya seedlings were inoculated to identify the transmission efficiency of each species of aphids and the experiment was repeated four times. As a control, 100 seedlings were inoculated using individual A. gossypii which were allowed to probe for $5 \mathrm{~min}$ on healthy papaya leaves. Test plants were transferred to a cage in an insect-proof glass house for symptom expression. After 30 days, the number of plants showing PRSV symptoms was recorded. Apical leaves were subjected to DAC-ELISA to further confirm PRSV infection. The percentage transmission data were subjected to ANOVA (at $P \leq 0.05$ significance) after arcsine transformation to compare the transmission efficiency of each aphid species.

Group inoculation. Groups of five A. gossypii alates starved for $2 \mathrm{~h}$ were given access to a PRSV infected source leaf for 5 min AAP and transferred to each of 25 healthy papaya seedling for an IAP of $5 \mathrm{~min}$. The experiment was repeated using two other species of aphid vectors (A. craccivora and $M$. persicae). Another set of 25 seedlings on which groups of 5 A. gossypii fed only on healthy leaves were placed for $5 \mathrm{~min}$ IAP, served as negative controls. After 30 days, symptoms were recorded and leaves were collected from each individual plant. Apical leaves were subjected to DAC-ELISA for confirmation of PRSV infection.

Duration of PRSV retention. To determine the duration of PRSV retention in aphid vectors, individual aphids from the three species A. gossypii, A. craccivora, and $M$. persicae, were given AAP to a PRSV source leaf for 5 min. Following AAP, aphids were placed in a plastic container for $5,10,30$, or 60 min without providing food (sequestration). After the appropriate sequestration period, 40 individual aphids from each group were allowed an IAP of 5 min on healthy papaya seedlings ( 1 aphid per seedling; 10 seedlings per replication; 4 replications). After 30 days, the number of plants showing PRSV symptoms was recorded. Apical leaves were subjected to DAC-ELISA to further confirm PRSV infection. Data were subjected to two factors ANOVA to compare the PRSV transmission efficiency of three aphid species following different sequestration periods.

Leaf-disk assay. A leaf-disk assay standardized for studying Tomato spotted wilt virus (TSWV) transmission by thrips (21) was suitably modified for aphid transmission studies. Instead of whole plants, leaf disks ( $3 \mathrm{~cm}$ diameter) from healthy plants were used for aphid transmission. Individual aphids were given an AAP of 5 min to a PRSV infected source leaf and an IAP of 5 min on a detached leaf from a healthy seedling. Following IAP, the probed area was marked and a circular disk was cut leaving the probed area at the center. Fifty leaf disks were used for each transmission experiment (five replications; 10 leaf disks per replication). After AAP and IAP, individual leaf disks were transferred to a petri dish containing fresh water. As controls, 50 individual $A$. gossypii were given 5 min AAP to a healthy leaf and then transferred to a healthy test leaf for 5 min of IAP. Leaf disks were retained on the water surface for 7 days. DAC-ELISA was carried out by macerating leaf disks and percent transmission was calculated. Data were subjected to ANOVA after arcsine transformation.

Sequential PRSV inoculation using leaf-disk bioassay method. The experiment was designed to determine the number of plants an individual viruliferous aphid can sequentially inoculate PRSV to. Ten individual A. gossypii, A. craccivora, and $M$. persicae were given access (AAP of $5 \mathrm{~min}$ ) to a PRSV infected leaf and then transferred to a healthy leaf for IAP. For the IAP, a single probe or a maximum of 5 min was allowed, then the aphid was transferred to a new, healthy leaf. The duration of the probe was recorded for each IAP. A few individual aphids continued to probe the leaf for $>5 \mathrm{~min}$ and had to be mechanically disturbed and transferred onto another leaf for the subsequent IAP. The process was repeated until five leaves (5 IAPs) had been subjected to sequential inoculation by each aphid. The experiment was repeated four times for each species of aphid studied. The leaf disks consisting of a probed area at the center were cut and floated on water. After 7 days, leaf disks were subjected to DACELISA and percent transmission for each IAP sequence was recorded. Data were subjected to two factors ANOVA to compare sequential PRSV inoculation efficiency in the three aphid vector species.

\section{RESULTS}

Measurement of vector efficiency. Single aphid inoculation. PRSV transmission efficiency differed significantly among the three aphid species (Table 1). M. persicae (56\%) followed by $A$. gossypii $(53 \%)$, were the most efficient vectors. A. craccivora (38\%) transmitted PRSV less efficiently compared to A. gossypii and $M$. persicae. A total of 44,42 , and $23 \%$ of plants showed characteristic PRSV symptoms 30 days after single-aphid inoculation by $M$. persicae, A. gossypii, and A. craccivora, respectively. All the plants expressing characteristic symptoms of PRSV were ELISA positive. However, ELISA detected PRSV infection in 12, 11 , and 15 additional plants inoculated with $M$. persicae, $A$. gossypii, and A. craccivora, respectively, indicating some of the plants were in fact infected, but did not express characteristic symptoms even 30 days after inoculation. The mean ELISA values were consistent for the 25 samples selected at random from each of the three species (Table 1). None of the 100 control plants on which individual A. gossypii were allowed an IAP of $5 \mathrm{~min}$ following an AAP on a healthy papaya leaf, showed PRSV symptoms and were negative by DAC-ELISA.

Significant differences in the time taken for the initiation of the first probe on the inoculation test plants were observed among the three species of aphids (Table 1). M. persicae would initiate probes in a significantly shorter period $(1.81 \pm 0.96 \mathrm{~min})$ followed by $A$. gossypii $(2.07 \pm 1.24 \mathrm{~min})$ and $A$. craccivora $(2.70 \pm$ $1.41 \mathrm{~min})$.

Group aphid inoculation. Contrary to results obtained through single-aphid inoculation, PRSV transmission efficiency of all three species increased to one hundred percent when a group of five aphids were used. All 25 plants showed characteristic symptoms 30 days after group aphid inoculation. PRSV was also detected by DAC-ELISA in all 25 plants inoculated using each species.

Duration of PRSV retention. There was a significant decrease in PRSV transmission with increased sequestration periods in all three species of aphids. Significantly higher transmission occurred 
when aphids were given IAP immediately following acquisition (no sequestration). After $5 \mathrm{~min}$ of sequestration, A. gossypii, A. craccivora, and $M$. persicae transmitted PRSV to $17.50,12.50$, and $22.50 \%$ of plants, respectively (Table 2). A few M. persicae could transmit PRSV even $30 \mathrm{~min}$ after AAP, but with significantly reduced efficiency (5\%) of transmission. No PRSV transmission was observed when A. gossypii and A. craccivora were sequestered for $30 \mathrm{~min}$ following AAP.

Leaf-disk bio-assay for measurement of vector efficiency. Transmission studies carried out using the leaf-disk bio-assay indicated that this assay could be successfully used for virus transmission by aphids in a nonpersistent mode instead of using the whole plant. Using this assay we could corroborate our earlier findings that individual $M$. persicae and $A$. gossypii were more efficient vectors compared to $A$. craccivora $(P \leq 0.05)$. Out of 50 leaf disks inoculated, PRSV was detected in 28, 24, and 15 leaf disks using ELISA after 7 days constituting 56, 48, and $30 \%$ transmission by $M$. persicae, A. gossypii, and A. craccivora, respectively (Table 3 ). Mean ELISA values of 15 PRSV positive $A$. craccivora inoculated samples were statistically on par with those from $M$. persicae and A. gossypii.

Sequential aphid inoculation. Following one AAP on PRSV infected papaya leaves, the three species of aphids varied in their ability to transmit to a series of leaf disks. Typically, the first or second transmission series (I or II IAP) was successful, but not always (Table 4). Individual $A$. gossypii and $M$. persicae were significantly more efficient than $A$. craccivora during the first series, with $M$. persicae being the most efficient at $52.50 \%$ transmission. However, transmission efficiency significantly decreased with the second and subsequent series (Table 4). A small percentage of A. gossypii could still transmit PRSV to the fourth leaf-disk in a series, but with reduced efficiency $(2.50 \%$ transmission). A. craccivora could transmit only up to the third series $(5.0 \%)$ and $M$. persicae only to the second (10\%).
The mean duration of the first probing on each test leaf-disk varied with the aphid species. A shorter probing period (1.86 s) was recorded for $A$. gossypii when transferred to leaf disks in the first and subsequent serial IAP (Table 5). This was followed by $A$. craccivora and $M$. persicae which probed for 2.37 and $2.99 \mathrm{~s}$, respectively. At each point in the series, $M$. persicae was observed to probe for a relatively longer period. The interaction effect between aphid species and duration of probing was significant $(P \leq 0.05)$ indicating distinct differences among the aphid species in the duration of probing at each inoculation series (at each IAP).

\section{DISCUSSION}

Single-aphid inoculation experiments indicated that all three aphid vectors (9) A. gossypii, A. craccivora, and M. persicae

TABLE 3. Transmission efficiency of three aphid species using a leaf-disk assay

\begin{tabular}{lccc}
\hline Aphid species & $\begin{array}{c}\text { Number of } \\
\text { leaf disks } \\
\text { inoculated }^{\mathrm{w}}\end{array}$ & $\begin{array}{c}\text { \% PRSV } \\
\text { transmission }^{\mathrm{x}}\end{array}$ & $\begin{array}{c}\text { Mean ELISA values } \\
\text { of 15 PRSV +ve } \\
\text { samples }\end{array}$ \\
\hline Aphis gossypii & 50 & $48.00(43.68) \mathrm{a}$ & $1.01 \pm 0.22$ \\
Aphis craccivora & 50 & $30.00(31.75) \mathrm{b}$ & $1.02+0.19$ \\
Myzus persicae & 50 & $56.00(45.00) \mathrm{a}$ & $1.14 \pm 0.20$ \\
Control & 50 & $0.00(0.00) \mathrm{c}$ & - \\
$F$ test & & *z & $\mathrm{NS}$ \\
CV\% & & 17.71 & 19.48 \\
SEM $( \pm)$ & & 4.40 & 0.17 \\
CD at $P \leq 0.05$ & & 9.55 & 0.36 \\
\hline
\end{tabular}

${ }^{\mathrm{w}}$ Five replications; 10 leaf disks per replication.

${ }^{\mathrm{x}}$ Numbers in parentheses are arcsine-transformed values; values followed by the same letter are not significantly different.

${ }^{y}$ Aphis gossypii were allowed to probe on healthy leaf instead of virus source leaf.

z Significant at $P \leq 0.05$.

TABLE 1. Comparison of transmission efficiency of Papaya ringspot virus (PRSV) by Aphis gossypii, A. craccivora, and Myzus persicae ${ }^{\mathrm{u}}$

\begin{tabular}{|c|c|c|c|c|c|}
\hline Aphid species & $\begin{array}{l}\text { Total number of } \\
\text { plants inoculated }\end{array}$ & $\begin{array}{l}\text { Mean time spent for initiation of first } \\
\text { probe on inoculation test plant (minutes) }\end{array}$ & 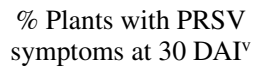 & $\begin{array}{l}\% \text { PRSV infection } \\
\text { confirmed by ELISA }^{\mathrm{w}}\end{array}$ & $\begin{array}{l}\text { Mean ELISA values of } \\
25 \text { PRSV +ve samples }\end{array}$ \\
\hline Aphis gossypii & 100 & $2.07 \pm 1.24 \mathrm{~b}$ & 42.00 & $53.00(46.75) \mathrm{a}$ & $1.59 \pm 0.21$ \\
\hline Aphis craccivora & 100 & $2.70 \pm 1.41 \mathrm{c}$ & 23.00 & $38.00(38.03) b$ & $1.59 \pm 0.15$ \\
\hline Myzus persicae & 100 & $1.81 \pm 0.96 \mathrm{a}$ & 44.00 & $56.00(48.48) \mathrm{a}$ & $1.66 \pm 0.19$ \\
\hline Control $^{\mathrm{x}}$ & 100 & - & 0.00 & $0.00(0.00) \mathrm{c}$ & - \\
\hline$F$ test & & $* \mathrm{y}$ & & $*$ & $\mathrm{NS}^{\mathrm{z}}$ \\
\hline CV $(\%)$ & & 53.75 & & 11.43 & \\
\hline $\operatorname{SEM}( \pm)$ & & 0.06 & & 3.11 & \\
\hline $\mathrm{CD}$ at $P=0.05$ & & 0.12 & & 7.02 & \\
\hline
\end{tabular}

u Individual aphid inoculation to each test plant; four replications; 25 seedlings per replication.

${ }^{\vee}$ Days after aphid inoculation.

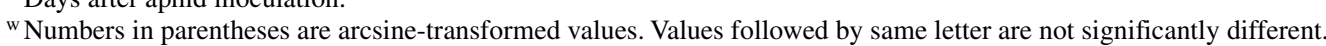

${ }^{x}$ Aphis gossypii were allowed to probe on inoculation test seedlings after probing on healthy leaf.

y Significant at $P \leq 0.05$.

${ }^{\mathrm{z}} \mathrm{NS}=$ nonsignificant.

TABLE 2. Duration of Papaya ringspot virus (PRSV) retention by three species of aphids ${ }^{2}$

\begin{tabular}{|c|c|c|c|c|}
\hline \multirow[b]{2}{*}{ Sequestration period (minutes) } & \multicolumn{3}{|c|}{$\%$ PRSV transmission } & \multirow[b]{2}{*}{ Mean } \\
\hline & Aphis gossypii & A. craccivora & Myzus persicae & \\
\hline 0 & $37.50(37.66)$ & $22.50(28.22)$ & $40.00(39.17)$ & $33.33(35.01) \mathrm{a}$ \\
\hline 5 & $17.50(24.53)$ & $12.50(17.89)$ & $22.50(28.22)$ & $17.50(23.54) b$ \\
\hline 30 & $0.00(0.00)$ & $0.00(0.00)$ & $5.00(9.22)$ & $1.66(3.07) \mathrm{cd}$ \\
\hline 60 & $0.00(0.00)$ & $0.00(0.00)$ & $0.00(0.00)$ & $0.00(0.00) \mathrm{d}$ \\
\hline Mean & $11.50(13.36) b$ & $7.50(10.14) \mathrm{c}$ & $15.50(18.49) \mathrm{a}$ & \\
\hline Duration & * & 2.70 & 5.45 & \\
\hline Interaction & NS & - & - & \\
\hline
\end{tabular}

${ }^{\mathrm{z}}$ Four replications; 10 seedlings per replication; NS = nonsignificant; numbers in parentheses are arcsine-transformed values. * indicates significant at $P \leq 0.05$. 
could successfully transmit the south Indian isolate of PRSV. No significant difference was observed in transmission efficiency between $M$. persicae and $A$. gossypii. These two predominant species (8) transmitted PRSV more efficiently compared to $A$. craccivora. Even though no published information is available on transmission efficiency of PRSV by different species of aphids, a number of workers have reported variation in aphid vector efficiency in transmitting other nonpersistent plant viruses $(18,19)$. Probing behavior could be one of the reasons for their variation in transmission efficiency in the three species. Our results showed that $M$. persicae and A. gossypii needed significantly less time for initiation of the first probe on inoculation test plants compared to A. craccivora. It is not very clear if this small yet significant difference would increase PRSV transmission in M. persicae and A. gossypii over that of A. craccivora. Time taken for the initiation of the first probe on the inoculation test plant influencing the efficiency of nonpersistent virus transmission by aphid vectors has been reported $(7,18,23)$. Nonpersistent viruses once acquired bind to the proximal tip of aphid stylets and are released when salivation occurs (3). Further, virus bound to these stylets is likely to be exposed to different enzymes and ionic conditions that may affect the surface structure of virions (3). Similarly, the chemical makeup of salivary secretion may vary with the vector species or even among biotypes within a species (3). These differences might have contributed to variation in PRSV transmission efficiency across three species of aphid vectors.

PRSV transmission efficiency of all three species of aphid vectors increased to one hundred percent when a group of five aphids was used for transmission in contrast to single-aphid inoculation (38 to $56 \%$ transmission). Often, under field conditions, $100 \%$ of papaya plants are PRSV infected, possibly because the same plant is inoculated by $>1$ aphid vector several times during its life span of 2 to 3 years. Nonpersistent viruses are transmitted rapidly to a number of plants in a manner related to the number of viruliferous vectors available (3). Further, one of the reasons for the lack of infection in some plants when individual aphids were used for transmission is possibly because certain individuals within an aphid species may not successfully acquire and transmit the virus. Similar observations were recorded in the western flower thrips, Frankliniella occidentalis (Pergande), where not all the individual thrips in a population were vectors of tomato spotted wilt virus (TSWV) (N. K. Krishna Kumar, A. E. Whitfield, and D. E. Ullman, unpublished data). At this stage, it is not clear whether this is true for aphid vectors that transmit viruses in a nonpersistent or persistent manner.

Following a single AAP, significant differences were observed among the three species of aphids in retaining PRSV and thereby influencing transmission efficiency. There was a step-wise reduction in PRSV transmission as the sequestration period increased. A. gossypii and A. craccivora lost their ability to transmit PRSV within 30 min of AAP even when they did not feed or probe on any leaf (though it is likely that a few viruliferous aphids could have probed on a plastic surface during sequestration). Similarly, A. gossypii and A. craccivora did not retain the zucchini yellow mosaic virus (a nonpersistent aphid-borne virus) beyond $1 \mathrm{~h}$ of sequestration (23). The receptors present in stylets may not hold the virus for a long period of time after acquisition and virions may find their way to the digestive tract, leading to a loss of their viruliferous nature $(12,13)$. Thus, the data suggest that after a prolonged sequestration period, the virus was either absent or was available, but no longer in a shape or form that was viable for transmission.

The results of the PRSV transmission during/following sequestration by different aphid species have epidemiological significance. PRSV infection has not been reported in the Coorg, North Kanara or South Kanara districts (parts of western ghat region shown as dotted area in Fig. 1) of Karnataka state $\left(15^{\circ} \mathrm{N} ; 75^{\circ} \mathrm{E}\right)$ nor did we observe a single PRSV infected plant in our repeated surveys, but severe incidence was noticed in the plains. The western ghat is a mountain range that runs along the western coast of India from Mumbai to the southern tip of the country for approximately $1,000 \mathrm{~km}$. There is a great variety of vegetation all along the ghat which includes scrub jungles, grassland along the lower altitudes, dry and moist deciduous forests, and semievergreen and evergreen forests. The plant diversity is high and papaya is not raised there as a monocrop in this region. Many cucurbitaceous vegetables are raised and $A$. gossypii is the major species colonizing these crops suggesting the presence of vectors and their host plants, but the absence of PRSV in this region. Thus, following PRSV acquisition, the probability of a viruliferous vector finding another papaya plant in a matter of $30 \mathrm{~min}$ to $1 \mathrm{~h}$ is very low. Almost all the major PRSV vectors are poly-

TABLE 5. Mean probing period (seconds) on each leaf disk (at each inoculation access period [IAP]) by three species of aphids in sequential inoculation experiments

\begin{tabular}{lccc}
\hline & \multicolumn{3}{c}{ Probing period on each leaf disk (seconds) } \\
\cline { 2 - 4 } IAPy & Aphis gossypii & A. craccivora & Myzus persicae \\
\hline I & 1.56 & 1.53 & 3.91 \\
II & 1.93 & 1.96 & 2.20 \\
III & 1.60 & 2.55 & 3.39 \\
IV & 1.88 & 3.30 & 2.33 \\
V & 2.31 & 2.53 & 3.11 \\
Mean & $1.86 \mathrm{a}$ & $2.37 \mathrm{~b}$ & $2.99 \mathrm{c}$ \\
& $F$ test & SEM $( \pm)$ & CD $(P \leq 0.05)$ \\
Aphid species & $* z$ & 0.25 & 0.50 \\
Interaction & $*$ & 0.57 & 1.12 \\
\hline
\end{tabular}

${ }^{\text {y }}$ Four replication; 10 alate aphids per replication.

z Significant at $P \leq 0.05$.

TABLE 4. Papaya ringspot virus (PRSV) transmission efficiency at each sequential inoculation by three species of aphids ${ }^{\mathrm{x}}$

\begin{tabular}{|c|c|c|c|c|}
\hline \multirow[b]{2}{*}{ IAPy } & \multicolumn{3}{|c|}{$\%$ Transmission } & \multirow[b]{2}{*}{ Mean } \\
\hline & Aphis gossypii & A. craccivora & Myzus persicae & \\
\hline I & $47.50(43.55)$ & $35.00(36.22)$ & $52.50(46.44)$ & $45.00(42.07) \mathrm{a}$ \\
\hline II & $32.50(34.71)$ & $25.00(29.88)$ & $10.00(15.86)$ & $22.50(26.82) b$ \\
\hline III & $15.00(22.50)$ & $5.00(9.22)$ & $0.00(0.00)$ & $6.67(10.57) c$ \\
\hline IV & $2.50(4.61)$ & $0.00(0.00)$ & $0.00(0.00)$ & $0.83(1.53) \mathrm{d}$ \\
\hline V & $0.00(0.00)$ & $0.00(0.00)$ & $0.00(0.00)$ & $0.00(0.00) \mathrm{d}$ \\
\hline \multirow[t]{2}{*}{ Mean } & $19.50(21.07) \mathrm{a}$ & $13.00(15.06) \mathrm{b}$ & $12.00(12.46) \mathrm{b}$ & \\
\hline & $F$ test & SEM $( \pm)$ & $\mathrm{CD}(P \leq 0.05)$ & \\
\hline Aphid species & $* \mathrm{z}$ & 1.69 & 3.46 & \\
\hline IAP & $*$ & 2.19 & 4.47 & \\
\hline Interaction & $*$ & 3.80 & 7.74 & \\
\hline
\end{tabular}

${ }^{\mathrm{x}}$ Four replications; 10 leaf disks per replication. Numbers in parentheses are arcsine-transformed values.

y Inoculation access period.

z Significant at $P \leq 0.05$. 
phagous and in the process of host selection, a vector could lose the virus inoculum. There is a very high probability of finding an appropriate host plant and colonizing it, thus reducing the chances of migration. Reduced vector pressure, high plant diversity, or lack of monoculture contributes to the absence of PRSV in Western ghats of Karnataka. This suggests that growing the main crop in the midst of nonhost crops (of virus) attenuates the spread of nonpersistent viruses.

The results of transmission efficiency studies using singleaphid inoculation to a series of five leaf disks indicated significant differences among the three species tested. No species transmitted PRSV to the fifth leaf disk (fifth sequence). A. gossypii was the most efficient species in transmitting PRSV to at least four successive leaf disks. However $M$. persicae, a more efficient vector at the first IAP, was least efficient in the second IAP. The data suggested that $A$. gossypii would be more efficient in transmitting PRSV to more plants in a papaya field than $M$. persicae or A. craccivora. This suggested A. gossypii (reported dominant species in terms of number) is also more efficient and can infect potentially four plants in some instances. This renders A. gossypii as the major vector responsible for large scale spread of PRSV in southern Karnataka.

Significant differences existed in the duration of probing by each species of aphids. A. gossypii probed on each leaf disk for a very short period, whereas $M$. persicae was observed to probe for longer duration. After acquiring the nonpersistent virus, aphids lose their ability to transmit the virus when they feed on the plants for a longer period $(13,15)$. Results of the present study also indicate that $A$. gossypii making short exploration and initiating probes was the most efficient vector. Our data supports the hypothesis that the durations of the probing and depth of the stylet probe has a drastic influence on vector efficiency (18). This may explain the vector efficiency of $A$. gossypii to transmit PRSV successfully to four leaf disks.

New PRSV infections were higher during April to May in southern Karnataka (8). During the papaya cropping period, the first peak of $A$. gossypii occurred during December to February (8). This peak may help to bring and introduce the virus from outside the field and infect some of the plants. Following this, a second vector peak was recorded during March to April. This peak activity of aphids is attributed to cropping patterns (especially large scale cultivation of cucurbitaceous crops), population density, and increased temperature (8). Hence, a high incidence of PRSV during April to May can be attributed to availability of virus source plants and increased activity of A. gossypii.

The results of the present study and our previous experiments suggested that monoculturing of papaya should be avoided, especially when adjacent crops or weed plants support enormous populations of any one of the vector species. Ensuring plant diversity either through mixed or inter-cropping will reduce the incidence of PRSV. Our observation supports the hypothesis provided by Hooks and Fereres (6) that flora diversification can reduce the incidence of many nonpersistent aphid-borne viruses. Hence, raising papaya as an intercrop or mixed crop could be one of the options for reducing PRSV incidence in endemic areas. Thus, considering the fluctuation in aphid vector activity and vector efficiency, planting of papaya (raised in an aphid-proof nursery) early in June (compared to the present practice of planting in August to September) in south Karnataka could reduce crop loss due to the lack of synchronization between peak activity of an efficient vector (A. gossypii) and availability of susceptible young seedlings. Studies on area-wise seasonal dynamics of different aphid species and their transmission efficiency is necessary before deploying any management strategies.

\section{ACKNOWLEDGMENTS}

We thank Director, IIHR for providing facilities to do this research. Our thanks to A. Vani and K. N. Chandrashekar, molecular diagnostic laboratory for technical help, Dr. Krishnareddy, virologist for help in identification of PRSV infected plants, A. Verghese, P. E. Rajashekaran, and R. Krishnan of IIHR for a critical review of manuscript. We also thank S. Joshi, Senior scientist, Project Directorate of Biological control, Bangalore for help in aphid identification. This work was financially supported by State Department of Horticulture, Government of Karnataka (HD/JDH/PLN/AHO-5/31/2004-05). This work is part of the Ph.D. thesis submitted by first author to UAS, GKVK, Bangalore.

\section{LITERATURE CITED}

1. Byadgi, A. S., Anahosur, K. H., and Kulkarni, M. S. 1995. Ringspot virus in papaya. The Hindu 118:28.

2. Gonsalves, D. 1994. Papaya ringspot virus. Pages 67-68 in: Compendium of Tropical Fruit Disease. R. C. Ploetz, G. A. Zentmyer, W. T. Nishijima, K. G. Rohrback, and H. D. Ohr, eds. Academic Press, New York.

3. Gray, S. M., and Banerjee, N. 1999. Mechanism of arthropod transmission of plant and animal viruses. Microbiol. Mol. Biol. R. 63:128-148.

4. Hema, M. V., and Prasad, D. T. 2004. Comparison of the coat protein of a South Indian strain of PRSV with other strains from different geographical locations. J. Plant Pathol. 86:35-42.

5. Hobbs, H. R., Reddy, D. V. R., Rajeshwari, R., and Reddy, A. S. 1987. Use of direct antigen coating and protein A coating ELISA procedures for detection of three peanut viruses. Plant Dis. 75:292-295.

6. Hooks, C. R. R., and Fereres, A. 2006. Protecting crops from nonpersistently aphid-transmitted viruses: A review on the use of barrier plants as a management tool. Virus Res. 120:1-16.

7. Kaakeh, W., and Dutcher, J. D. 1993. Population dynamics and probing behavior of cowpea aphid A. craccivora (Homoptera: Aphididae) on preferred and nonpreferred host cover crops. J. Entomol. Sci. 28:145-155.

8. Kalleshwaraswamy, C. M., Krishnakumar, N. K., Verghese, A., Dinesh, M. R., Ranganath, H. R., and Venugopalan, R. 2007. Role of transient aphid vectors on the temporal spread of papaya ringspot virus in south India. Acta Hortic. 740:251-258.

9. Khurana, S. M. P., and Bhargava, K. S. 1971. Three new vectors of papaya viruses. J. Hortic. Sci. 46:209-211.

10. Mora-Aguilera, G., Teliz, D., Campbell, C. L., and Avilla, C. 1992. Temporal and spatial development of papaya ringspot in Veracruz, Mexico. J. Phytopathol. 136:27-36.

11. Namba, R., and Higa, S. Y. 1981. Papaya mosaic transmission as affected by the duration of the acquisition probe of the green peach aphid - Myzus persicae (Sulzer). P. Hawaii Entomol. Soc. 23:431-443.

12. Nault, L. R. 1997. Arthropod transmission of plant viruses - A new synthesis. Ann. Entomol. Soc. Am. 90:521-541.

13. Ng, J. C. K., and Perry, K. L. 2004. Transmission of plant viruses by aphid vectors. Mol. Plant Pathol. 5:505-511.

14. Purcifull, D. E., Edwardson, J., Hiebert, E., and Gonsalves, D. 1984. Papaya ringspot virus. CMI/AAB Description of Plant viruses, No. 292.

15. Plumb, R. T., and Callow, J. A. 2002. Plant virus vector interactions. Page 220 in: Advances in Botonical Research. eds. Academic Press, New York.

16. Prasad, S. M., and Sarkar, D. P. 1989. Some ecological studies on Papaya ringspot virus in Ranchi. Indian J. Virol. 5:118-122.

17. Verghese, A., Anil Kumar, H. R., and Jayanthi, P. D. K. 2001. Status and possible management of papaya ringspot virus with special reference to insect vectors. Pest Manag. Hort. Ecosyst. 7:99-112.

18. Stubbs, L. L. 1960. Aphid transmission of broad bean wilt virus and comparative transmission efficiency of three vector species. Aust. J. Agr. Res. 11:734-741.

19. Summers, C. G., Newton, A. S., Jr., Kirk, M., and Temple, S. R. 1990. Transmission of beet yellow and beet mosaic viruses by non-colonizing aphid vectors. J. Econ. Entomol. 83:2448-2451.

20. Wang, H. L. 1981. Aphid transmission of Papaya ringspot virus in Taiwan. Plant Prot. Bull. 23:229-233.

21. Wijkamp, I., and Peters, D. 1993. Determination of median latent period of two tospoviruses in Frankliniella occidentalis, using a novel leaf disk assay. Phytopathology 83:986-991.

22. Yeh, S. D., Bau, H. J., Cheng, Y. H., Yu, T. A., and Yang, J. S. 1998. Greenhouse and field evaluation of coat protein transgenic papaya resistant to papaya ringspot virus. Acta Hort. 461:321-328.

23. Yuan, C., and Ullman, D. E. 1996. Comparison of efficiency and propensity as measures of vector importance in Zucchini yellow mosaic potyvirus transmission by Aphis gossypii and A. craccivora. Phytopathology 86:698-703. 